\title{
Disseminated Nocardiosis: The Complexity of the Diagnosis
}

\author{
Cristina Marques ${ }^{\mathrm{a}, \mathrm{b}}$, Marcia Ribeiro ${ }^{\mathrm{a}}$, Maria Teresa Bonccoraglio ${ }^{\mathrm{a}}$, \\ Joana Braga ${ }^{\mathrm{a}}$, Ana Francisca Pereira ${ }^{\mathrm{a}}$, Ana Ogando ${ }^{\mathrm{a}}$
}

\begin{abstract}
Nocardiosis is a rare infection in immunocompetent patients. Nocardia spp. is an uncommon cause of prostate abscesses and is responsible for only $1-2 \%$ of brain abscess. Hematogenous dissemination can occur, and presentation of abscesses in more than two locations is required to determine a disseminated nocardiosis. The microbiological diagnosis of this agent is still a challenge due to the complexity of its identification in regular laboratories. An early diagnosis and adequate treatment with effective antibiotics are critical for treating this entity. We report a case of a patient who presented with brain abscess with a previous medical history of prostate abscess to Nocardia spp. which evolved to disseminated nocardiosis.
\end{abstract}

Keywords: Nocardia spp.; Brain abscess; Disseminated nocardiosis; Immunocompetent

\section{Introduction}

Nocardia spp. is a kind of aerobic, gram-positive and weakly acid-fast bacteria that are commonly found in soil, water and plants [1, 2]. Infections by Nocardia spp. are rare in healthy individuals, but more common in immunocompromised patients [3]. Risk factors include long-term corticosteroid and other immunosuppressor therapies, malignancy, human immunodeficiency virus (HIV) infection, diabetes and alcoholism [4]. Infection by Nocardia usually occurs through direct cutaneous inoculation or inhalation but can spread hematogenously to the central nervous system (CNS), which is a severe form of the disease, presenting as brain abscess or meningitis that can manifest as seizures, headaches or focal neurological deficits [1]. The authors present a case of an immunocompetent patient who presented with a $\mathrm{No}$ cardia brain abscess evolving to disseminated nocardiosis.

Manuscript submitted February 2, 2021, accepted February 16, 2021

Published online March 5, 2021

anternal Medicine Department, Hospital Santa Maria Maior, Barcelos, Portugal

${ }^{\mathrm{b}}$ Corresponding Author: Cristina Isabel Moreira Marques, Internal Medicine Department, Hospital Santa Maria Maior, Barcelos, Portugal.

Email: Marques.cristinaa@gmail.com

doi: https://doi.org/10.14740/jmc3673

\section{Case Report}

A 78-year-old man presented to the emergency department (ED) after a seizure, altered mental state and mild cough. The patient's medical records showed a history of hypertension, dyslipidemia, alcohol consumption of $90 \mathrm{~g}$ per day and prostate abscess. Two months previously, the patient presented a prostate abscess being treated with drainage and antimicrobial therapy with ceftriaxone for 7 days. Moreover, physical exam revealed floating state of consciousness and a fever of $38.1^{\circ} \mathrm{C}$, with alterations in pulmonary and cardiac auscultation, and no focal neurological deficits.

Laboratory studies showed relative neutrophilia (neutrophils $9.5 \times 10^{9} / \mathrm{L}$ ), sedimentation rate of $40 \mathrm{~mm}$, slightly elevated C-reactive protein (CRP) levels $(13.80 \mathrm{mg} / \mathrm{L})$ and mild hyponatremia of $132 \mathrm{mmol} / \mathrm{L}$. A cerebral computed tomography $(\mathrm{CT})$ scan was performed showing "two expansive intraaxial areas, suggestive of lesions of a tumoral nature, probably secondary" (Fig. 1). Chest X-ray showed no signs of pneumonia. The patient was admitted to the infirmary with cerebral lesions of uncertain nature in study and a respiratory tract infection, being treated on amoxicillin-clavulanate $1.2 \mathrm{~g}$ every $8 \mathrm{~h}$.

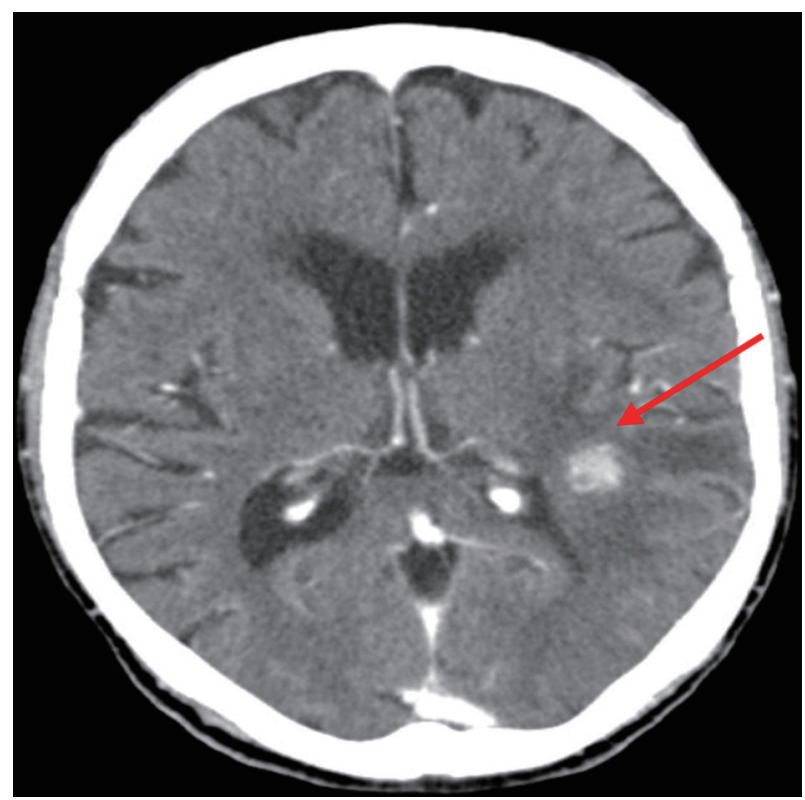

Figure 1. Admission cerebral computed tomography (CT) scan, showing a nodular lesion and surrounding edema (arrow). 


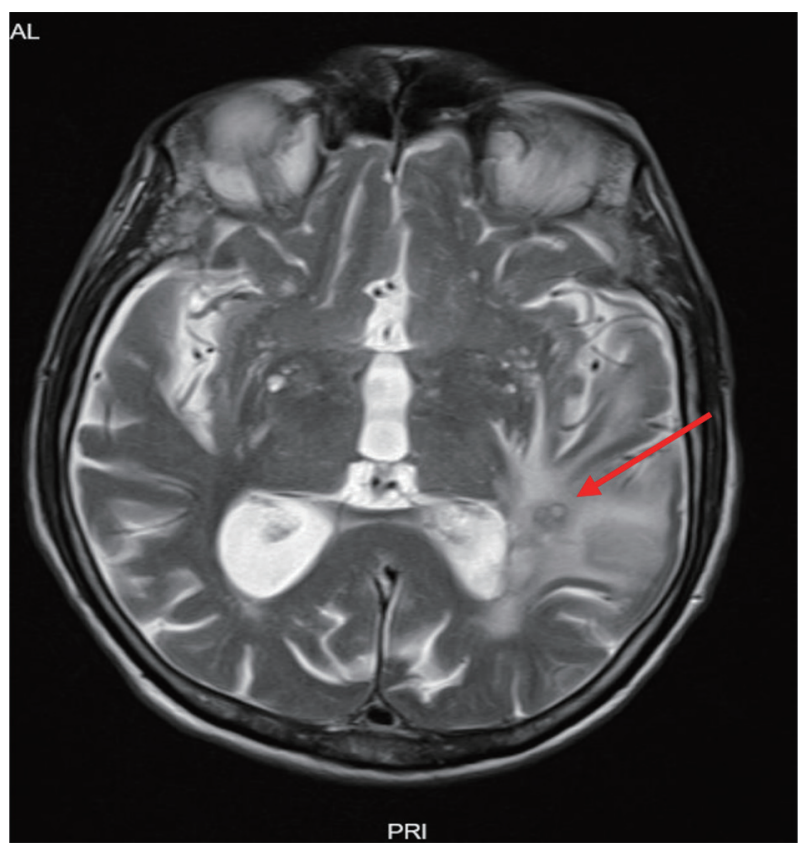

Figure 2. Magnetic resonance imaging (MRI) of brain T2 sequence, showing cerebral edema consisting of cerebritis and periventricular abscess (arrow).

Within 4 days of the admission, the patient had been submitted to multiple imaging tests, which included a thorax, abdominal and pelvic CT scan, a transthoracic echocardiogram, and an ear-throat-nose exam showing no signs of malignancy or other lesions. The presence of sustained fever and deterioration of the conscientious level suggested that those cerebral lesions might be due to infectious cause.

Empiric therapy with ceftriaxone $2 \mathrm{~g}$ every $12 \mathrm{~h}$ plus metronidazole $1 \mathrm{~g}$ every $12 \mathrm{~h}$ was started, bringing partial clinical improvement. A magnetic resonance imaging (MRI) of the brain was performed, showing "periventricular white matter abscesses, with deposit of infectious material in the lateral ventricles and apparent area of temporal cerebritis" (Fig. 2).

The complementary studies with HIV antibody, hepatitis B surface antigen and hepatitis $\mathrm{C}$ antibody were all negative

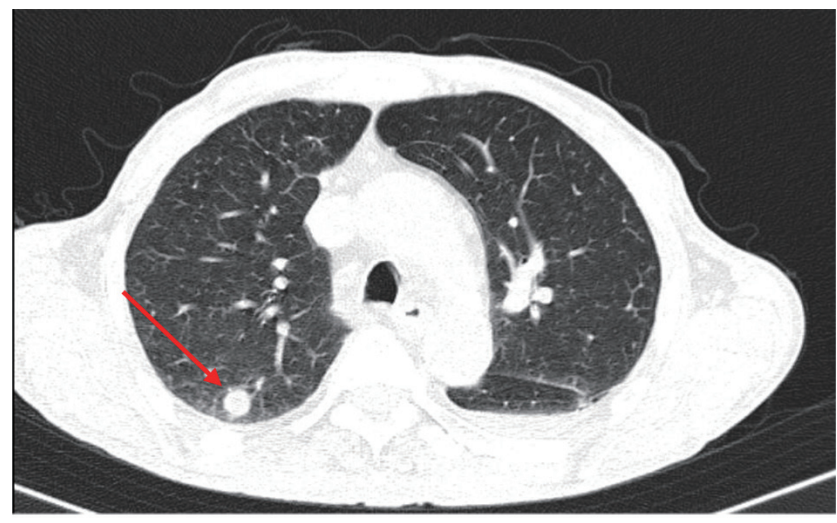

Figure 3. Computed tomography (CT) scan of thorax showing lung abscess (arrow).

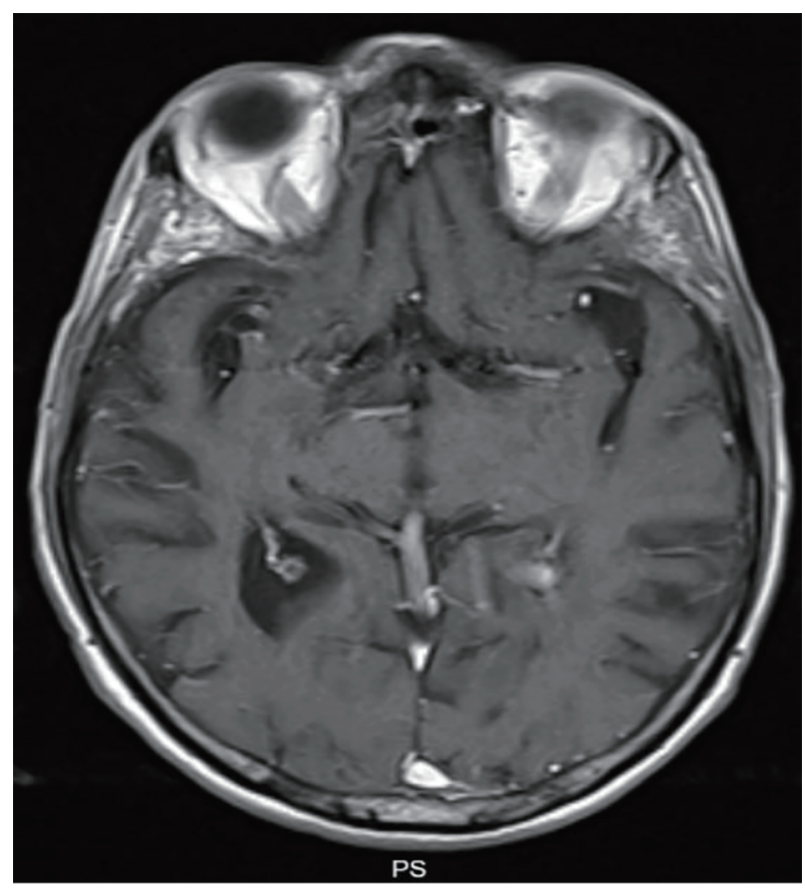

Figure 4. Magnetic resonance imaging (MRI) of brain-T1 sequence showing resolution of the lesions presented previously.

and immunoglobulins were within normal range. After reviewing the patient's clinical record, the microbiological result of the prostatic abscess pus showed Nocardia spp., which could explain the partial response to ceftriaxone. A multidisciplinary discussion determined that Nocardia spp. was the most likely agent responsible in the case. Empirical parenteral antibiotics were started with trimethoprim-sulfamethoxazole (TMP-SMX), amikacin and imipenem, as an antimicrobial susceptibility testing (AST) was not available, bringing clinical improvement.

At the 10th day of therapy, blood work showed a pancytopenia with severe neutropenia and TMP-SMX was suspended, with no recovery of white blood cells. A granulocyte colonystimulating factor (G-CSF) was prescribed with satisfactory response. After recovery of neutrophils, multiple small abscesses were found in lungs, liver, kidneys and spleen (Fig. 3). The patient went under multiple procedures, such as liver biopsy, bronchoalveolar lavage and repeated blood cultures, but isolation was never possible in the samples obtained.

Once the antibiotic therapy was adjusted, because of the side effects presented, it was concluded in a multidisciplinary discussion and with an infectious disease expert that our patient should undergo intravenous therapy with amikacin and imipenem until resolution of the lesions presented, which happened at 8 months of therapy (Fig. 4).

A consumption of alcohol of $90 \mathrm{~g}$ per day was the only cause of immunosuppression found and Nocardia spp. were only found in the prostate pus. Due to the severity and complexity of the disease, prostate abscess to Nocardia spp. with hematogenous dissemination to brain, lungs, liver, kidneys and spleen, after discussion with infectious diseases experts, it was decided to discharge the patient with ceftriaxone plus amoxi- 
cillin/clavulanate until completing 12 months of antimicrobial therapy. This treatment was supported by the AST of Nocardia spp. in the Iberian Peninsula and in the partial response obtained with ceftriaxone initially. There was full resolution of the multiple lesions and, after 12 months of follow-up, the patient remained with no signs of reappearance of the lesions.

\section{Discussion}

Nowadays, more than 50 Nocardia species were identified [5], and present as pulmonary or disseminated disease in more than $90 \%$ of cases [1]. In the Iberian Peninsula, the most common is Nocardia cryiacigeorgica [2]. Infections of the CNS by Nocardia spp. typically present as meningitis or sub-acute abscess and account for $1-2 \%$ of brain abscesses $[1,3,6]$, with a mortality rate of $20 \%$ in immunocompetent patients [1], higher in those immunocompromised patients or those with known risk factors $[1,7]$. The median incubation time to detect Nocardia bacteriemia is 3 - 5 days [4], and a specific ribosomal ribonucleic acid (rRNA) sequencing is needed for the identification of the species [7], which is a challenge in the diagnosis in many hospitals. Dual intravenous antimicrobial therapy is the initial treatment according to AST available for most species. TMP-SMX is the first-line therapy having a resistance rate of $11 \%$ and an association with amikacin and imipenem is preferred as those agents have a low rate of resistance, $2 \%$ and $15 \%$ respectively $[1,4,7]$. The usual duration of therapy is 6 months and can be extended up to 12 months depending on the severity of the disease [4]. A switch to oral agents after 3 - 6 weeks can occur depending on the evolution [7]. The choice of antimicrobials should be held on the regional AST [7].

In the case that we are discussing, there was evidence of Nocardia spp. in the prostate abscess pus. The patient did not presented risk factors to the first site infection (prostate biopsy, surgery, instrumentation of the urinary tract and urinary tract disorders) [7]. Hematogenous spread of the Nocardia spp. to brain was confirmed once the patient started to improve after 1 week of empiric aimed antibiotic therapy.

Severe neutropenia flourish most likely due to TMP-SMX. A treatment with G-CSF was required in order to normalize the leucocytes count. As a result, new abscesses in the lungs, liver, kidneys and spleen were found and represented a challenge when a switch to oral antibiotics was possible. Without the Nocardia spp. identification and AST, a dual antimicrobial therapy with ceftriaxone and amoxicillin/clavulanate was considered as the preferred one having the local AST in account.

\section{Conclusions}

In conclusion, nocardiosis is a rare entity and should not be overlooked in the differential diagnosis of infections as its incidence is increasing, being more common among immunocompromised patients, whose diagnosis is still a challenge. Early suspicion and diagnosis are required to prevent morbidity and mortality. This case illustrates the complexity and severity of this infection, its diagnostic approach and the need of an adequate treatment according to local AST when only Nocardia spp. was identified.

\section{Acknowledgments}

None to declare.

\section{Financial Disclosure}

All authors declare that no financial support was received from any organization for the submitted work.

\section{Conflict of Interest}

None to declare.

\section{Informed Consent}

Both written and verbal informed consent was obtained from the patient for publication of this case report.

\section{Author Contributions}

$\mathrm{CM}$ and $\mathrm{MR}$ are the physicians who worked with patient and contributed to manuscript writing; MTB, JB, AFP and AO are the physicians who worked with patient and were involved in manuscript review.

\section{Data Availability}

The authors declare that data supporting the findings of this study are available within the article.

\section{References}

1. Patel H, Patel B, Jadeja S, Isache C. Central nervous system nocardiosis masquerading as metastatic brain lesions. IDCases. 2019;18:e00652.

2. Leite IM, Trigueiros F, Martins AM, Fonseca M, Marques T. Disseminated nocardiosis: a case report. Cureus. 2019;11(8):e5294.

3. Kim CH, Lee H, Woo CG, Han JH, Choi H, Park SM. Primary pancreatic candidiasis mimicking pancreatic cancer in an immunocompetent patient. Korean J Gastroenterol. 2021;77(1):45-49.

4. Williams E, Jenney AW, Spelman DW. Nocardia bacteremia: A single-center retrospective review and a systematic review of the literature. Int J Infect Dis. 2020;92:197207.

5. Conville PS, Brown-Elliott BA, Smith T, Zelazny AM. The complexities of nocardia taxonomy and identifica- 
tion. J Clin Microbiol. 2018;56(1).

6. Zhu JW, Zhou H, Jia WQ, You J, Xu RX. A clinical case report of brain abscess caused by Nocardia brasiliensis in a non-immunocompromised patient and a relevant litera- ture review. BMC Infect Dis. 2020;20(1):328.

7. Sakamaki I, Ueno A, Kawasuji H, Miyajima Y, Kawago K, Hishikawa Y, Ikehata Y, et al. Prostate abscess caused by Nocardia farcina. IDCases. 2019;18:e0640. 